\title{
Dinámica de Fluidos Computacional de la Hidrólisis de Aceite de Soja Bajo Condiciones de Agua Subcrítica en un Reactor de Tanque Agitado
}

Andrés F. Aldana ${ }^{1 *}$, Johnnys A. Bustillo ${ }^{1}$, Juan C. Urueta ${ }^{2,3}$ y Antonio J. Bula ${ }^{1}$

(1) Universidad del Norte, Departamento de Ingeniería Mecánica, Barranquilla, Colombia

(e-mail: afaldana@uninorte.edu.co; bjohnnys@uninorte.edu.co; abula@uninorte.edu.co)

(2) Departamento de Innovación, PALMACEITE S.A., Santa Marta, Colombia

(e-mail: proyectos@aceitesa.com)

(3) Departamento de Innovación, ACEITES S.A., Santa Marta, Colombia

Recibido Feb. 21, 2018; Aceptado May. 3, 2018; Versión final Jun. 18, 2018, Publicado Oct. 2018

\begin{abstract}
Resumen
Se determina mediante el uso de herramientas computacionales el comportamiento de la hidrólisis del aceite de soja con agua subcrítica. Empleando la cinética de reacción de segundo orden propuesta en la literatura, se realiza una simulación fluido dinámica empleando el programa COMSOL MULTIPHYSICS. Se utiliza un enfoque $2 \mathrm{D}$ en un plano horizontal que representa la sección transversal de un reactor agitado. Se consideraron los efectos turbulentos y se seleccionaron los modelos TRANS y $\mathrm{k}-\varepsilon$ para las simulaciones, que se desarrollaron a tres niveles de calentamiento (523K, $548 \mathrm{~K}$ y $573 \mathrm{~K})$. Los resultados obtenidos a $548 \mathrm{~K}$ fueron consecuentes con los datos experimentales referenciados, presentando un error aproximado de $4 \%$ con lo reportado.
\end{abstract}

Palabras clave: hidrólisis de aceite; agua subcrítica; reacción autocatalítica; dinámica computacional de fluidos

\section{Computational Fluid Dynamics of the Soybean Oil Hydrolysis under Subcritical Water Conditions in a Stirred-Tank Reactor}

\begin{abstract}
Using computational tools, the behavior of soybean oil hydrolysis under subcritical conditions was predicted. Using a second order kinetic model proposed in the literature, computational fluid dynamics simulation was coupled with the reaction set using COMSOL MULTIPHYSICS. A 2D approach over the horizontal plane representing the reactor cross section was applied. Turbulence effects were included using TRANS and $K-\varepsilon$ models. Three different temperature levels $(523 \mathrm{~K}, 548 \mathrm{~K}$ and $573 \mathrm{~K})$ were simulated. The results for $548 \mathrm{~K}$ represented properly the referred experimental data with an approximated absolute error of $4 \%$.
\end{abstract}

Keywords: oil hydrolysis; subcritical water; autocatalytic reaction systems; computer fluid dynamics; turbulent heat and mass transfer 


\section{INTRODUCCIÓN}

El agua subcrítica -SW- se ha convertido en una de las sustancias más interesantes para realizar la hidrólisis de lípidos. Estudios recientes han explorado el uso de este fluido en diferentes biomasas como paja de trigo (Abdelmoez et al., 2014), algas (Reddy et al., 2014) y aceites vegetales como girasol (Alenezi et al., 2009), soja (Schall et al., 2013) o maíz (Pinto y Lancas, 2006). El agua subcrítica también ha mostrado ventajas significativas en los procesos de extracción de aceite, según Quin et al. (2015) los tiempos se pueden reducir ocho veces en comparación con la extracción por destilación al vapor y también el rendimiento aumenta aproximadamente un $21.5 \%$ en comparación con el mismo método. En la actualidad, el procesamiento subcrítico de lípidos ha sido estudiado a escala de laboratorio por diversos autores, algunos como, Abdelmoez et al. (2014) utilizó un sistema continuo en reactores tubulares, mientras que Reddy et al. (2014) utilizó un reactor de microondas, en cambio Alenezi et al. (2009) utilizó un CSTR y Schall et al. (2013) realizaron el proceso en un tanque agitado por lotes. Debido a la naturaleza del proceso, se dispone de datos cinéticos limitados. Pramote et al. (2004) indica que la hidrólisis en SW de ésteres de ácidos grasos en un reactor discontinuo responde a una cinética de primer orden, (Schall et al., 2013) proporcionó un mecanismo dividido compuesto mediante un conjunto de reacciones secuenciales de primer orden y un segundo conjunto de reacciones autocatalíticas. La experimentación a escala de laboratorio implica el uso de equipos especializados que operan a alta presión y temperatura, lo que representa que el uso de herramientas de simulación como CFD podrían ayudar a aumentar las capacidades de investigación en este campo utilizando los datos experimentales realmente obtenidos como medida de comparación. Wang et al. (2012) realizó una simulación de CFD de hidrólisis de aceite de canola.

En el análisis de un reactor de tanque agitado, la utilización de herramientas como el CFD se ha utilizado ampliamente para analizar la hidrodinámica, las propiedades de mezcla y la cinética de reacción química de varios procesos. Rudniak et al. (2004) realizó un análisis CFD para sistemas de reacción homogéneos y heterogéneos utilizando un enfoque marco euleriano-euleriano; mientras que Baldyga et al. (2011) lo usa para establecer el efecto de la mezcla en reacciones químicas paralelas y (Hongna et al., 2014) modelan un sistema gas-líquido en un reactor de tanque agitado de múltiples fases.

En este trabajo, el modelado CFD se utiliza para simular la hidrólisis del aceite de soja de acuerdo con la cinética expresada por (Schall et al., 2013), utilizando el programa COMSOL MULTIPHYSICS en OD y 2D obteniendo un rendimiento proyectado y estableciendo qué zonas de la sección transversal tienen diferencias en la concentración de la especie y la temperatura de la mezcla.

\section{DESCRIPCIÓN DEL FENÓMENO}

La descripción del fenómeno se presenta en 4 subsecciones: se inicia con una descripción de las características generales del aceite de soja y del agua subcrítica, luego se describen lo referente a la reacción de hidrólisis utilizada.

\section{Características del Aceite de Soja}

El contenido de aceite en las semillas de soja normalmente varía entre el 19 y el $21 \%$ en peso (Dijkstra, 2016) y comercialmente está disponible en tres tipos, crudo desgomado, una vez refinado y completamente refinado; y dependiendo del tipo, se aplica un estándar específico. El perfil particular de ácidos grasos para el aceite de soja se muestra en la Tabla 1 (Erhan y Perez, 2002).

Tabla 1: Perfil de ácidos grasos para el aceite de soja

\begin{tabular}{cccccc}
\hline Sustancia & Palmítico (16:0) & Esteárico (18:0) & Oleico (18:1) & Linoleico (18:2) & Linolénico (18:3) \\
\hline Aceite de Soja & 6.0 & 5.2 & 20.2 & 63.7 & 5.0 \\
\hline
\end{tabular}

\section{Características del Agua subcrítica}

Se considera que el agua está en estado subcrítico cuando se encuentra en estado líquido entre los $100^{\circ} \mathrm{C}$ y $374^{\circ} \mathrm{C}$, en ese intervalo los enlaces de hidrógeno debido a la agitación térmica se debilitan, lo que genera una disminución significativa en la constante dieléctrica del fluido, aumentando así la solubilidad de compuestos normalmente hidrofóbicos tales como lípidos y aromáticos (Carr et al., 2011). La figura 1 ilustra el comportamiento de esta propiedad a diferentes temperaturas de agua líquida saturada. El aumento de la solubilidad del compuesto hidrófobo en aguas subcríticas se debe a la reducción de estas propiedades (Plaza y Turner, 2015), a la capacidad de donación de enlaces de hidrógeno (acidez), capacidad de aceptación de enlaces de hidrógeno (basicidad) y polaridad. 


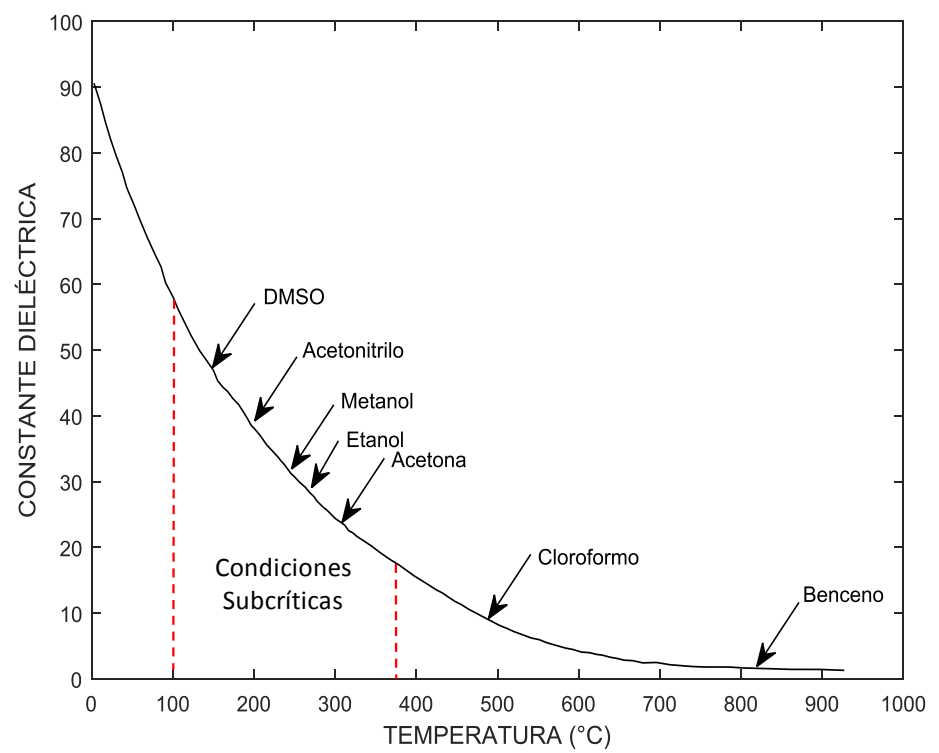

Fig. 1: Comparación de la constante dieléctrica sensible a la temperatura del agua a presión de líquido saturado con las constantes dieléctricas de diferentes solventes a temperatura ambiente (Adaptada de Carr et al., 2011)

La densidad del líquido, la conductividad térmica y el calor específico de la fracción lipídica de aceite de soja se pueden calcular de acuerdo con las siguientes expresiones (Barbosa-Canovas y lbarz, 2002). En la figura 2 (Ting y Chen, 2011) muestra la viscosidad del aceite y el impacto de la temperatura sobre esta.

$$
\begin{aligned}
& \rho=9.2559 \times 10^{-2}-0.41757 * T \\
& k=0.18071+2.7604 \times 10^{-3} * T-1.7749 \times 10^{-7} * T^{2} \\
& C_{P}=1.9842+1.4733 \times 10^{-3} * T-4.8008 \times 10^{-6} * T^{2}
\end{aligned}
$$

\section{Reacción de hidrólisis de aceite}

El aceite de soja puede considerarse como una mezcla predominante de ésteres de ácido carboxílico y agua. La hidrólisis de este tipo de material ha sido ampliamente estudiada y el mecanismo general de reacción se muestra en la ecuación 4 (Wolfe y Jerrers, 2000):

$$
\mathrm{R}-\mathrm{COO}-\mathrm{R}+\mathrm{OH}^{-} \rightarrow \mathrm{R}-\mathrm{COOH}+\mathrm{HOR}
$$

\section{Cinética para la hidrólisis de aceite con agua subcrítica}

(Alenezi et al., 2009) descubrió que el rendimiento de ácidos grasos en una hidrólisis de aceite de girasol con agua subcrítica describía una función sigmoidal que sugería involucrar un fenómeno autocatalítico; de acuerdo con eso (Schall et al., 2013) formuló el siguiente sistema de reacción:

$$
\begin{aligned}
& T G+W \leftrightarrow D G+F A \\
& D G+W \leftrightarrow M G+F A \\
& M G+W \leftrightarrow G+F A \\
& T G+F A+W \leftrightarrow D G+2 F A \\
& D G+F A+W \leftrightarrow M G+2 F A \\
& M G+F A+W \leftrightarrow G+2 F A
\end{aligned}
$$

Las ecuaciones 5 a 7 describen el conjunto estándar de reacciones del sistema, mientras que las ecuaciones 8 a 10 representan las reacciones autocatalíticas del fenómeno. (Diasakou et al., 1998) encontraron que la sección estándar de las reacciones en un fenómeno de transesterificación mostró tasas de reacción similares, por lo que (Schall et al., 2013) sugirió acoplar las velocidades cinéticas de las ecuaciones 5 a 10 en dos grupos, uno para el estándar y un segundo valor para la sección autocatalíticas suponiendo una dependencia de Arrhenius sobre la temperatura. La Tabla 2 muestra los parámetros cinéticos del sistema. En la tabla 2, las unidades del factor de frecuencia son $\left(\mathrm{m}^{3} / \mathrm{s} \mathrm{mol}\right)$ para la sección estándar $\left(\mathrm{m}^{6} / \mathrm{s}^{2} \mathrm{~mol}\right)$ y para la parte Autocatalítica del sistema. 


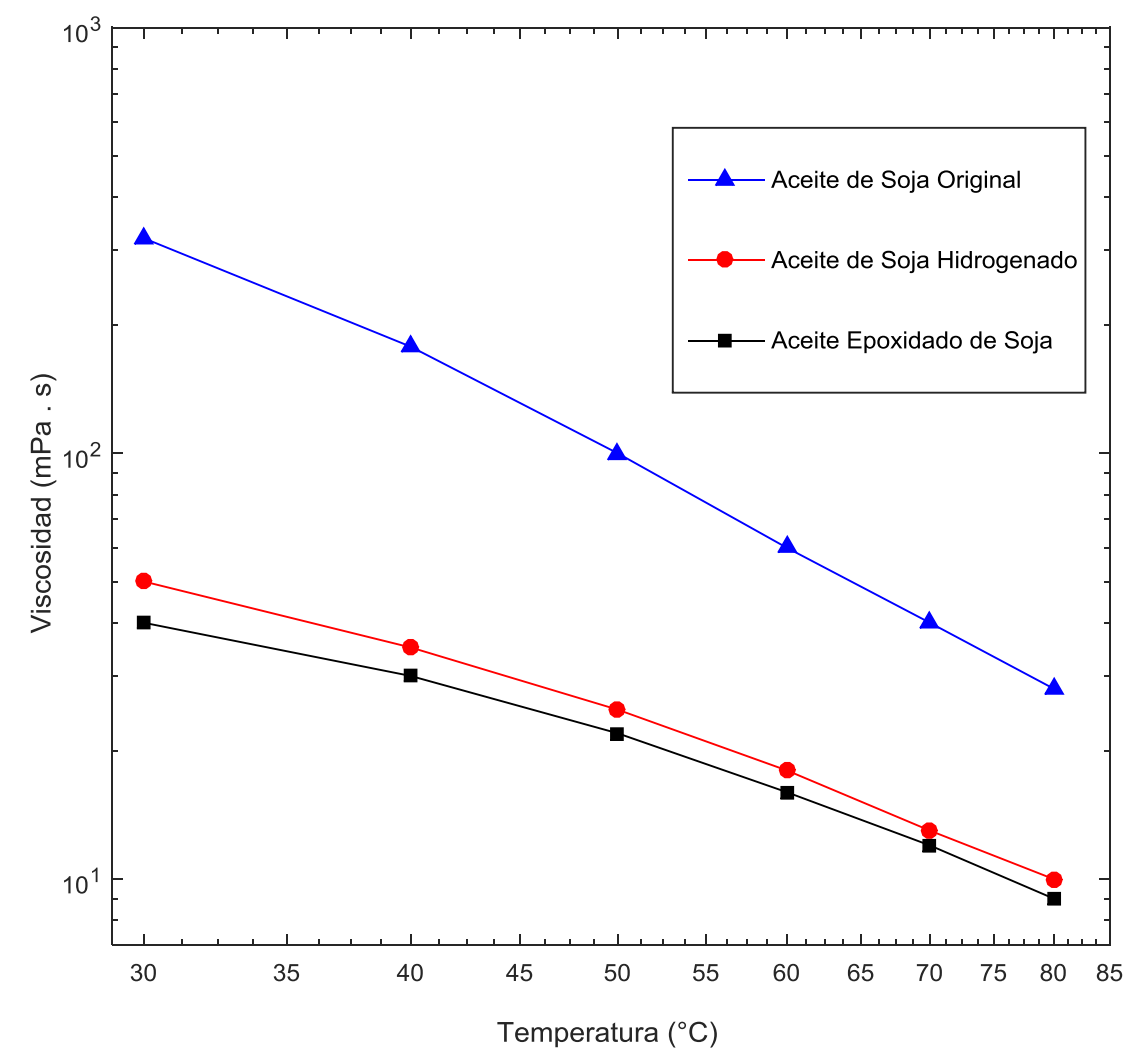

Fig. 2: Efecto de la temperatura en la viscosidad de varios aceites de soja (Ting y Chen, 2011)

Tabla 2: Parámetros cinéticos para el Sistema de reacciones

\begin{tabular}{cccc}
\hline Sección & Dirección & $\begin{array}{c}\text { Factor de } \\
\text { Frecuencia }\end{array}$ & $\begin{array}{c}\text { Energía de Activación } \\
\mathrm{J} / \mathrm{mol}\end{array}$ \\
\hline Estándar & Hacia adelante & 3.91 & 90,290 \\
Estándar & Reversa & $1.2937 \times 10^{8}$ & 158,750 \\
Autocatalítica & Hacia adelante & $2.6414 \times 10^{-8}$ & 26,770 \\
Autocatalítica & Reversa & 0.875 & 95,230 \\
\hline
\end{tabular}

\section{METODOLOGÍA}

La simulación puede verse como una copia de una situación real, en los procesos de ingeniería química esta característica es extremadamente importante para adquirir conocimiento de un proceso complejo (Bandyopadhyay, 2014). En este caso se utiliza el programa COMSOL MULTIPHYSICS para tal fin. El módulo de ingeniería química se seleccionó para simular el comportamiento del sistema de reacción basado en la cinética descrita anteriormente en un reactor agitado por lotes, buscando establecer si el comportamiento de variables objetivo como el rendimiento de conversión y la concentración de especies son homogéneos dentro del equipo o hay zonas con mayor eficiencia en el dominio estudiado. La figura 3 ilustra la metodología aplicada.

\section{Descripción del proceso}

La simulación se configuró asumiendo un reactor discontinuo de acero inoxidable de $250 \mathrm{ml}$ (dimensiones Amar Equipments PVT Modelo AC2503392) y método de calentamiento (figura 4), se asumió una velocidad de agitación de 700 rpm y se estableció una proporción aceite / agua de 1: 4 y un 14\% en peso de la cantidad total de carga del reactor de ácido linoleico se consideró agregado como catalizador, luego la agitación comienza a desarrollar un patrón de flujo y finalmente se activa la fuente de calentamiento suponiendo una temperatura constante en todo el calentador. El tiempo de simulación fue de 40 minutos y el experimento computacional se realizó con tres niveles de temperatura $(523,548$ y $573 \mathrm{~K})$ para establecer líneas base de comparación con el experimento (Schall et al., 2013). 


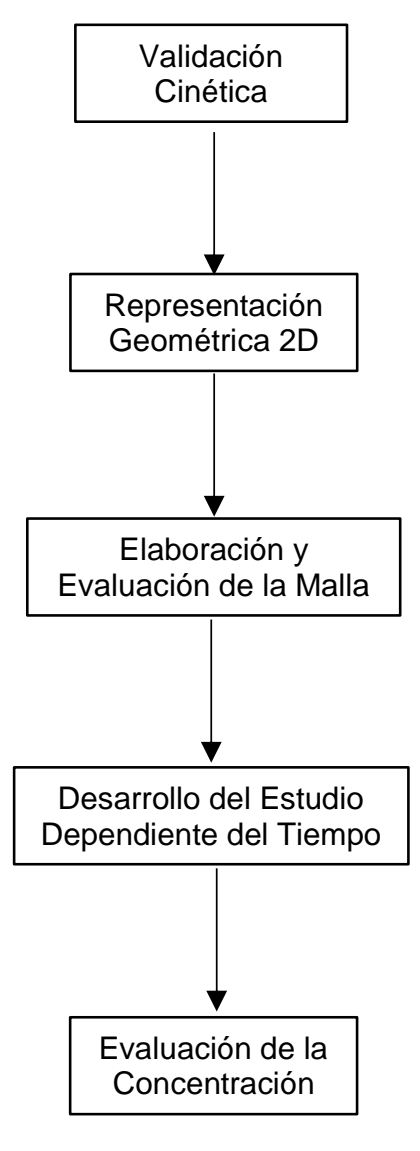

Fig. 3: Metodología

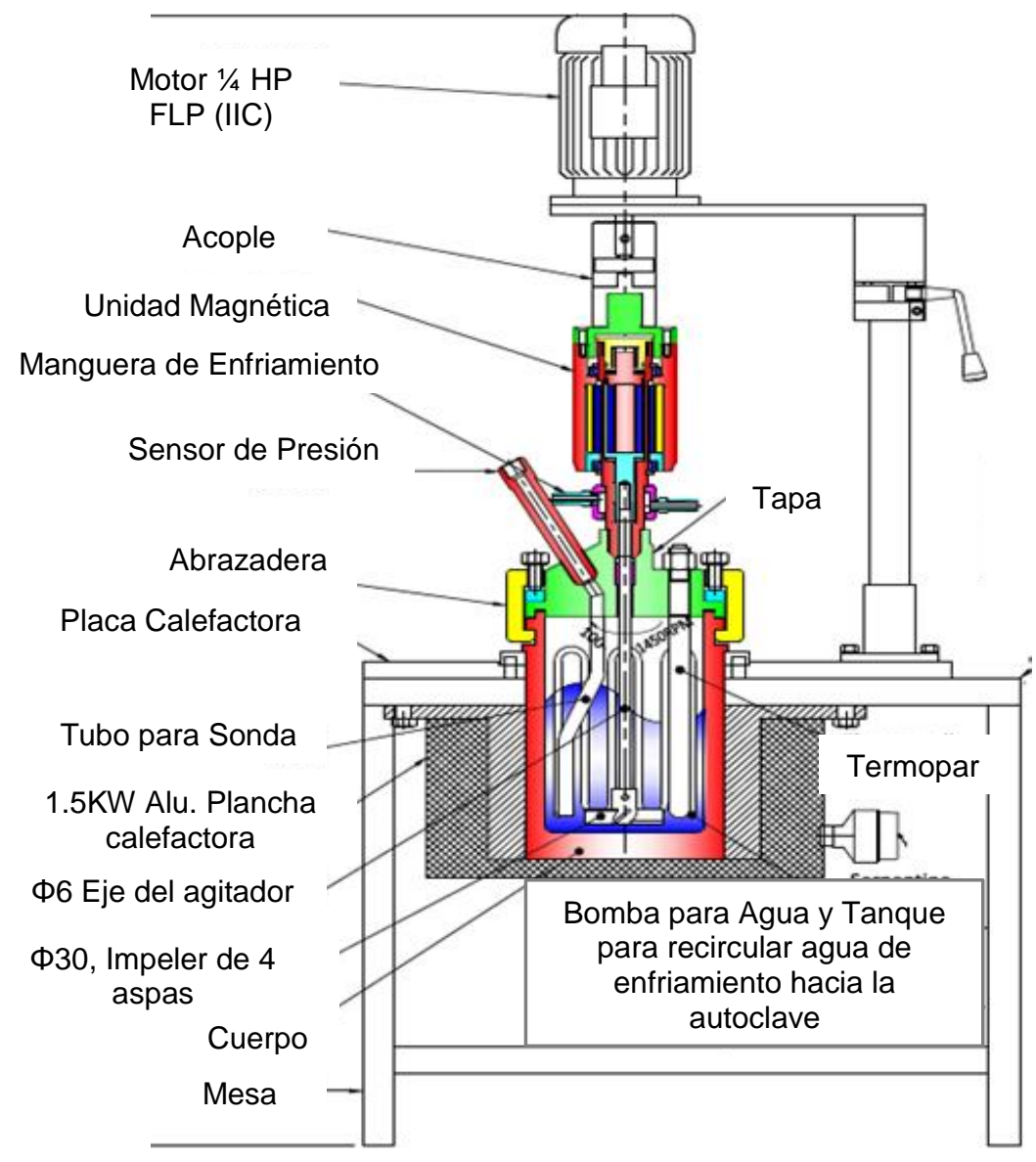

Fig. 4: Plano del reactor utilizado en la simulación

\section{RESULTADOS}

A continuación, se presentan los resultados obtenidos de forma separada para los perfiles de concentración de especies, evaluación y convergencia del mallado, perfiles de concentración de ácidos grasos obtenidos y el perfil de distribución de la temperatura.

\section{Validación de la cinética: experimentos en $O D$}

Después de la configuración computacional con la cinética de reacción establecida en la subsección de cinética, se realizó un estudio adimensional suponiendo una mezcla perfecta. La concentración de especies, la temperatura de mezcla y el rendimiento de ácidos grasos (FA) se establecieron como variables objetivo y en este caso se simuló un tiempo de proceso de $100 \mathrm{~min}(6000 \mathrm{~s})$ a $523 \mathrm{~K}, 548 \mathrm{~K}$ y $573 \mathrm{~K}$.

Las figuras 5,6 y 7 muestran los perfiles de concentración de especies y de temperatura respectivamente para cada nivel de temperatura. Los resultados obtenidos en la concentración de especies muestran una influencia positiva.

Las figuras 5, 6 y 7 muestran que la producción de ácido graso se ve favorecida con temperaturas de reacción más altas, esto podría verse comparando los intervalos de concentración de FA que son significativamente mayores a $573 \mathrm{~K}$; también la cinética de reacción alcanza la estabilidad más rápido debido a la aparición más temprana del punto de inflexión que indica un período de estabilización más largo a temperaturas más altas, evaluando este criterio se pudo observar que a $573 \mathrm{~K}$ el punto de inflexión aparece casi $10 \mathrm{~min}$ antes en comparación con 523K.

Además, la figura 8 confirma el impacto positivo que tuvieron temperaturas de reacción más altas en el sistema. La caída de temperatura fue 4 grados menor a 573K que 523K, y la estabilización de temperatura fue más rápida a temperaturas iniciales más altas. 


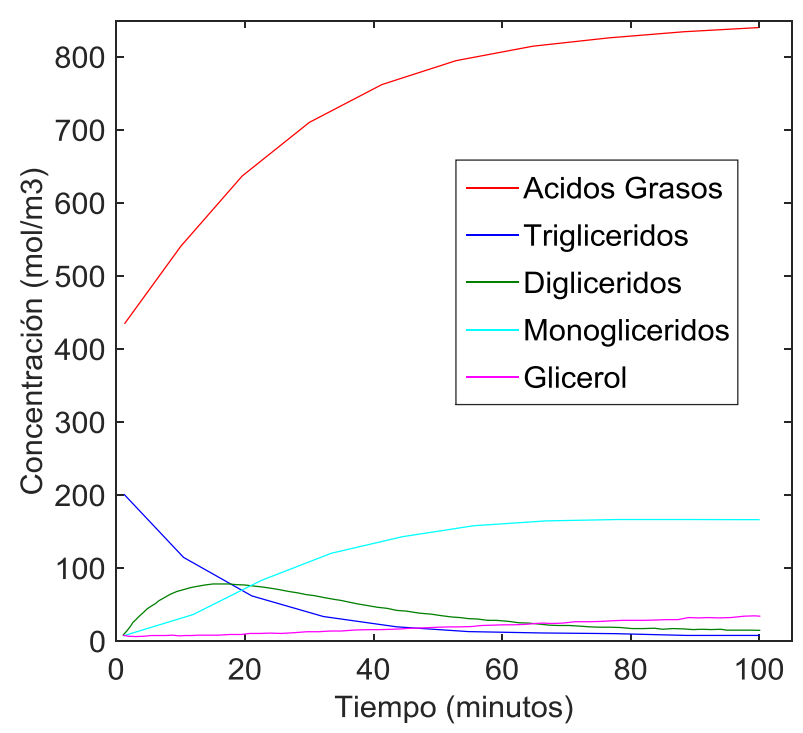

Fig. 5: Perfil de concentración de especies para 523K

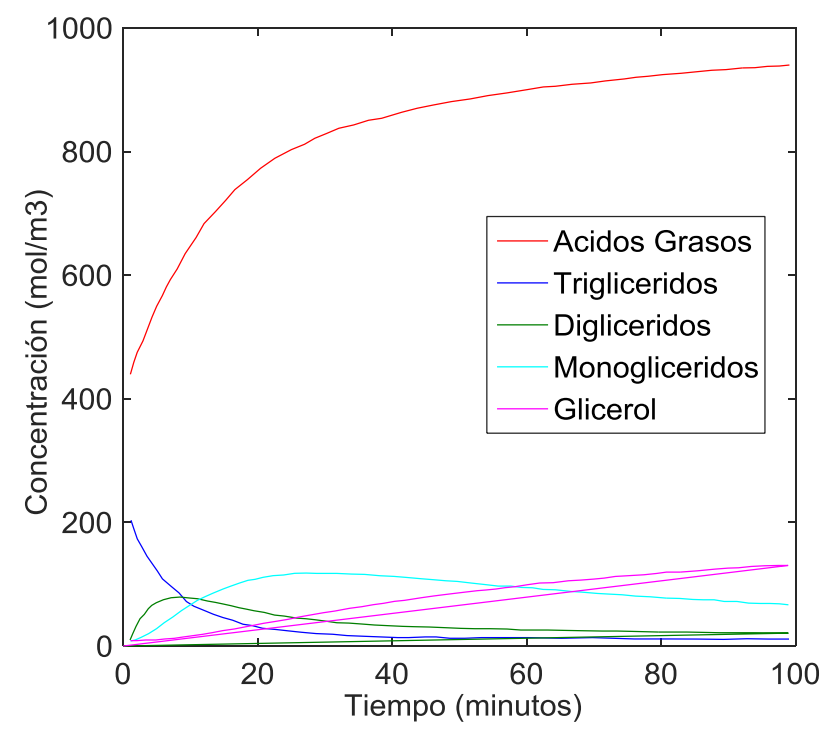

Fig. 7: Perfil de concentración de especies para 573K

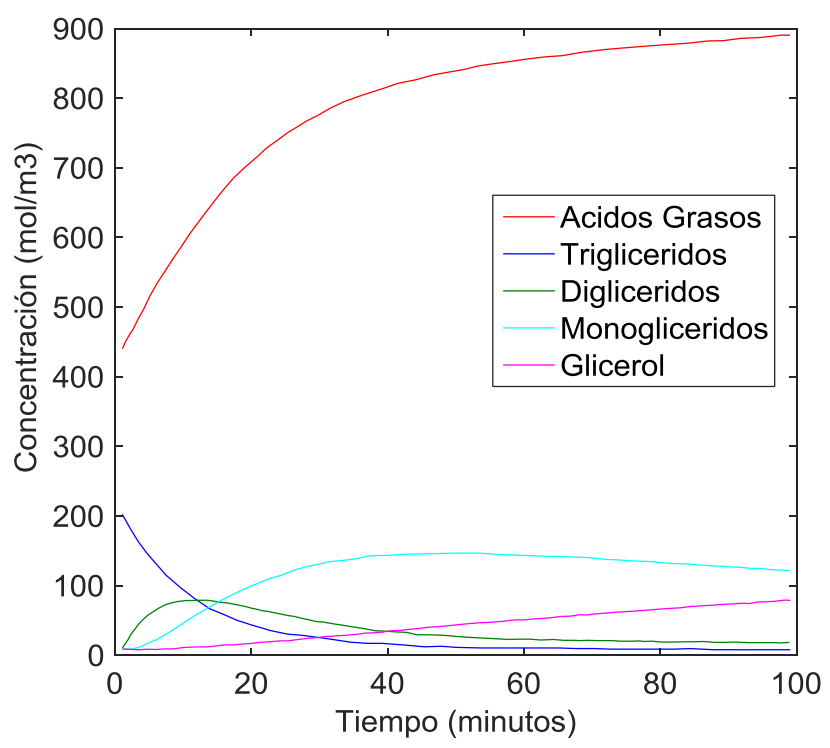

Fig. 6: Perfil de concentración de especies para 548K

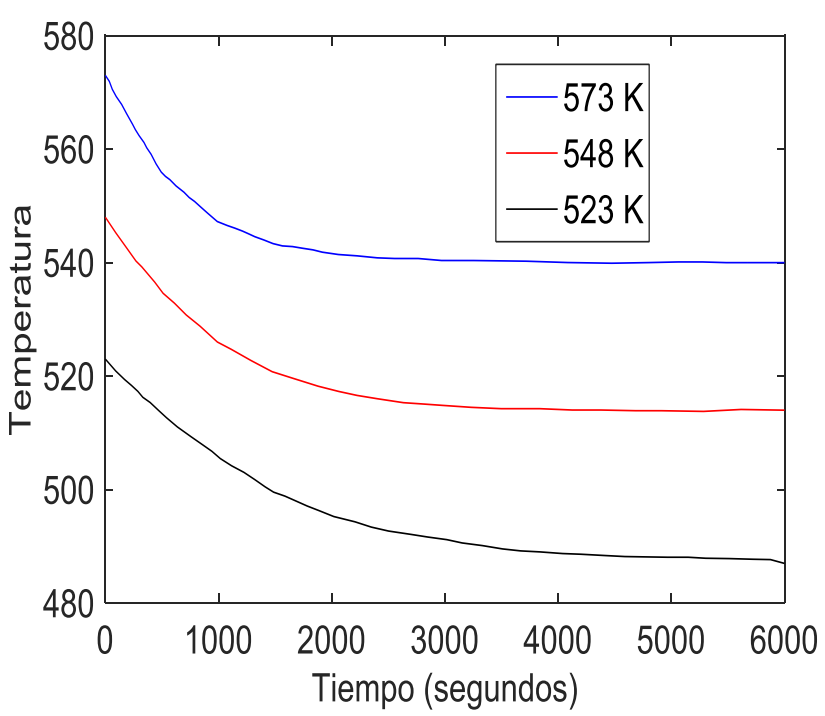

Fig. 8: Perfil de temperatura de la mezcla

Una vez establecido los perfiles de concentración de especies a las temperaturas de trabajo se procedió a establecer el rendimiento estimado de la operación, para esto se comparó el resultado de concentración de ácidos grasos obtenida contra la concentración máxima teórica, determinada utilizando las ecuaciones 5, 6, 7,8, 9 y 10. La expresión que ilustra dicha relación se muestra en la ecuación 11.

$$
Y_{F A(t)}=\frac{C_{F A(t)}-C_{F A(i)}}{3 C_{T G(i)}}
$$

La figura 9 muestra el rendimiento de FA obtenido para cada temperatura de reacción y confirma el impacto positivo de llevar la reacción a temperaturas más altas, mostrando un rendimiento de conversión cerca del $15 \%$ mayor a $573 \mathrm{~K}$ que el obtenido a $523 \mathrm{~K}$ después de $100 \mathrm{~min}$ de proceso (entre $0.8 \mathrm{~mol} \mathrm{~mol}^{-1}$ y $0.6 \mathrm{~mol}$ $\left.\mathrm{mol}^{-1}\right)$.

También se puede apreciar que en cada uno de los niveles de temperatura se muestra un comportamiento logarítmico entre el rendimiento y la temperatura en el tiempo. Alcanzando un punto de inflexión a partir de los 1500 segundos de proceso. 


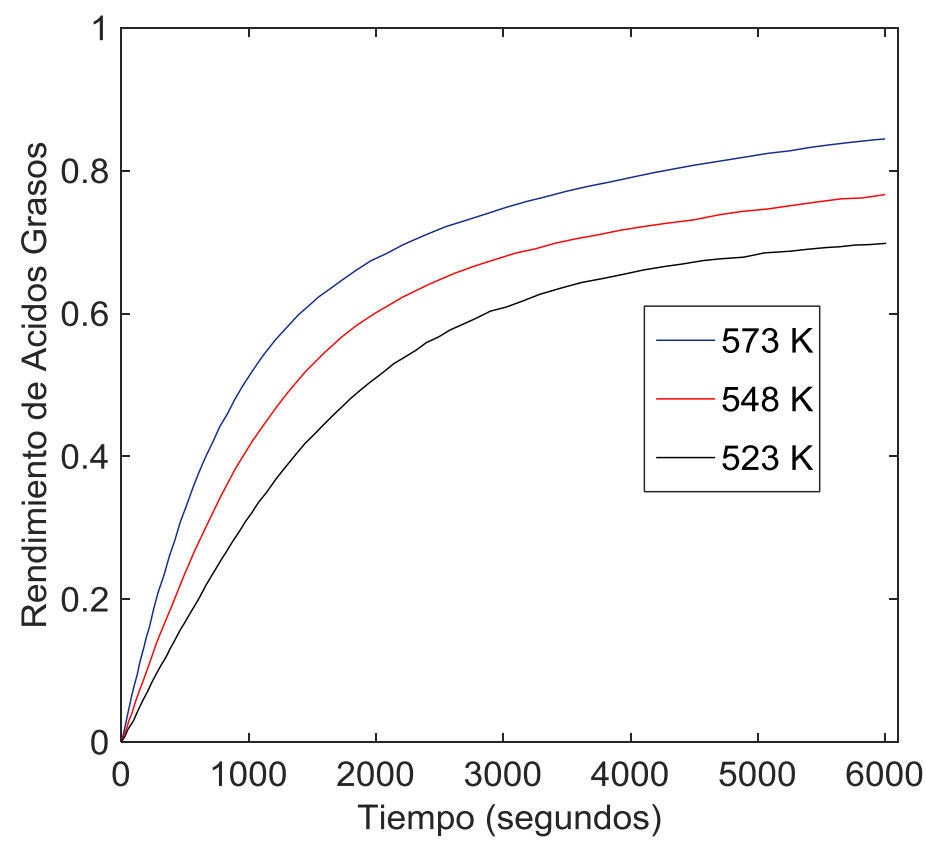

Fig. 9: Perfil de ácidos grasos obtenidos para 523K, 548K y 573K

\section{Representación bidimensional de la geometría}

El fenómeno se representará como una sección transversal del tanque considerado, la figura 10 muestra una ilustración de la geometría utilizada.

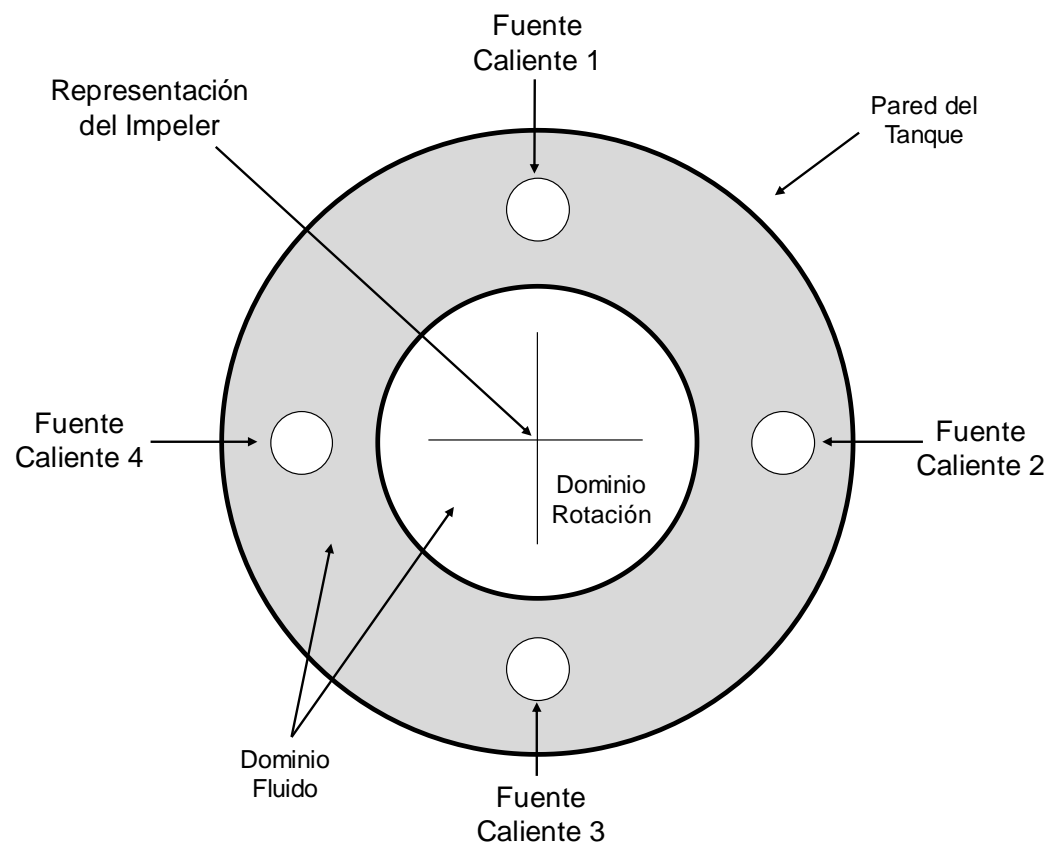

Fig. 10: Geometría utilizada para la simulación

\section{Elaboración y evaluación de la malla}

En esta sección se presenta con detalle la generación de la malla y el análisis de independencia de la malla con el fin de determinar cuál de las mallas generadas fue adecuada para el estudio

Generación de la malla: En la figura 10, se generó una malla heterogénea con una técnica de descomposición de dominio (Conelly y Kokini, 2007) estableciendo un marco giratorio central y un marco inercial donde las ecuaciones de momento se resuelven ordinariamente. Además de los marcos de referencia múltiples, también debido a la naturaleza del fenómeno, el refinamiento de la malla se aplicó en las zonas críticas de la geometría (Impulsor, fuentes de calor y dominio giratorio) y se aplicó relajación en el dominio de la pared del tanque. Tres mallas fueron generadas y las figuras 11,12 y 13 los ilustran. 


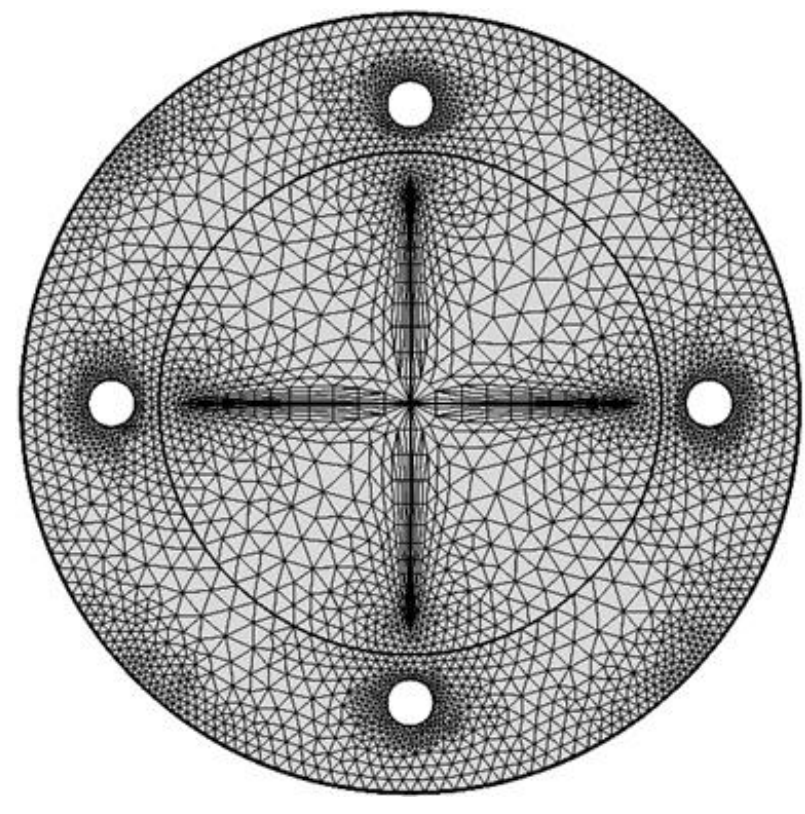

Fig 11: Mallado \# 1; 7,630 elements

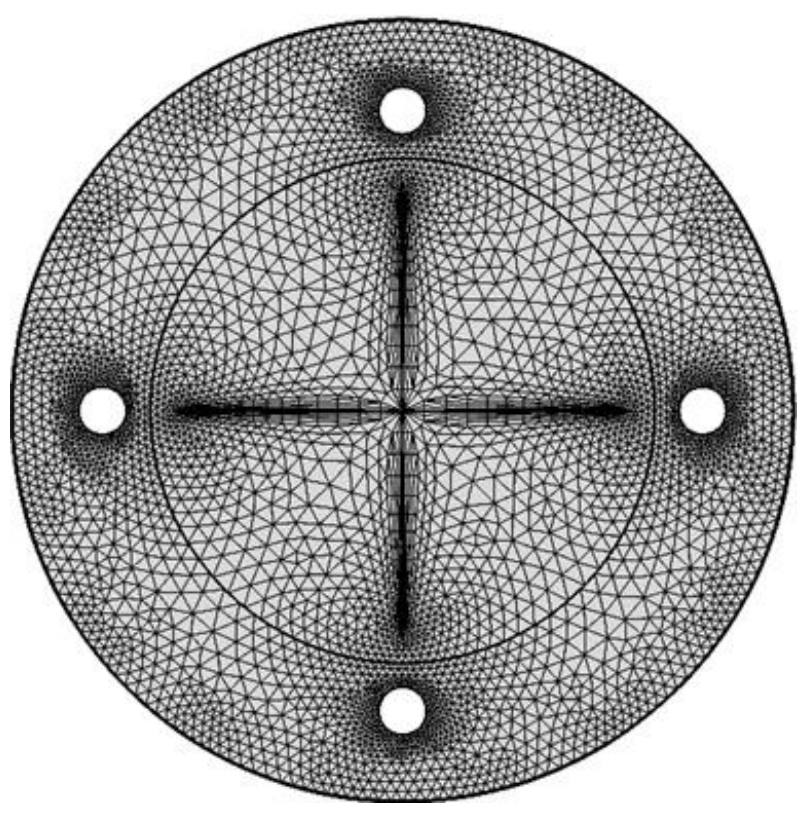

Fig 12: Mallado \# 2; 9,967 elements

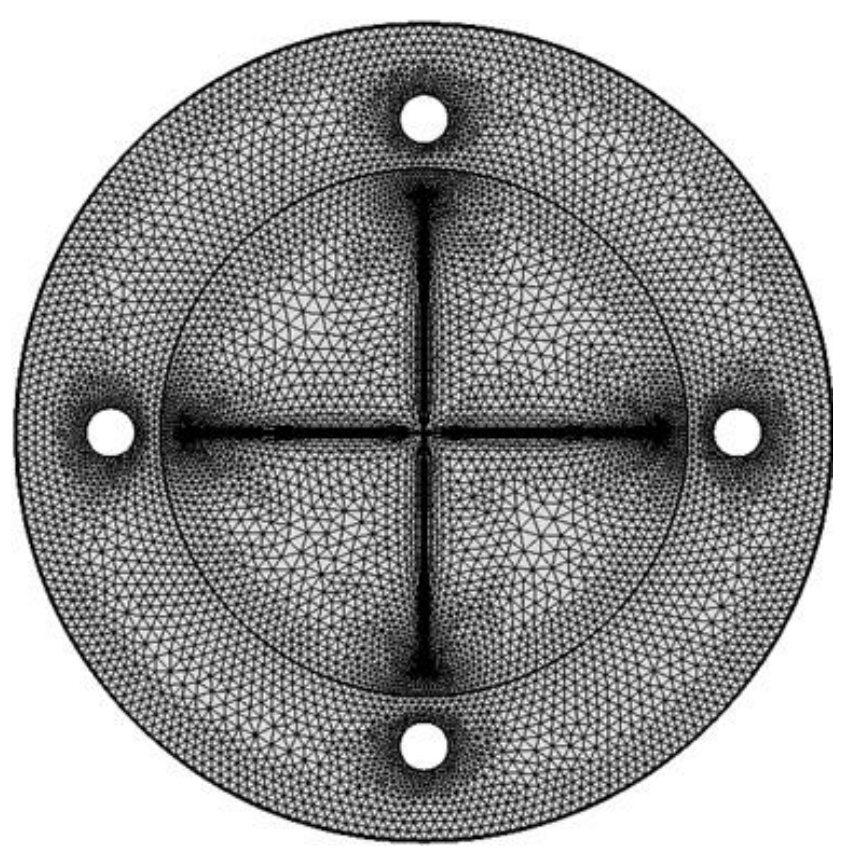

Fig 13: Mallado \# 3; 16,402 elements

Independencia de la malla: Para determinar cuál de las mallas generadas fue adecuada para el estudio, se realizó un ejercicio de simulación de mezcla de estado estacionario con la velocidad absoluta como variable objetivo. La evaluación se basó usando los resultados de 33 puntos comunes en el sistema entre mallas y comparación gráfica de los perfiles de velocidad obtenidos mostrados en la figura 14. La evaluación visual mostró ligeros cambios en los sectores de alta velocidad del perfil principalmente en Malla \#1. La velocidad absoluta de puntos comunes se muestra en la figura 15 confirmando que los resultados entre la malla \#2 y \#3. están más cerca en comparación con la malla \#1. El índice de selección de malla se establece de acuerdo con la siguiente expresión:

$$
M S_{I}=\frac{U_{M(i)}-U_{M(i-1)}}{E_{M(i)}-E_{M(i-1)}}
$$

Donde:

$M S_{I}=$ Indice de selección de malla, $U_{M}=$ Velocidad y $E_{M}=$ Cantidad de elementos en la malla 
En la ecuación (12) los valores de $M S_{I(1 \rightarrow 2)}$ y $M S_{I(2 \rightarrow 3)}$ son $1.98 \times 10^{-6}$ y $2.17 \times 10^{-7}$ mostrando que el cambio de la malla \#2 a la malla \#3 tiene diez veces menos impacto que pasa de una malla \#1 a una \#2. De acuerdo con este criterio, la malla \#2 fue seleccionada.

\section{Índice de Convergencia del Mallado}

El nivel de independencia del mallado se evalúa utilizando la extrapolación de Richardson (Roache, 1997) a partir de tres soluciones. La Tabla 3 muestra los cálculos respectivos.

Tabla 3: Evaluación del índice de Convergencia de Malla (GCI)

\begin{tabular}{ccccccccc}
\hline$h$ & $f$ & $r$ & $e$ & $G C l$ & f.exact & $p$ & Fs & \\
\hline 4 & 0.694929098 & & & & & & & \\
2 & 0.699550154 & 2 & $0.661 \%$ & $0.357 \%$ & 0.702 & 1.727 & 1.25 & 0.998 \\
1 & 0.698154107 & 2 & $0.200 \%$ & $0.108 \%$ & 0.698 & & & \\
\hline
\end{tabular}

El resultado del análisis es 0.998 , lo que es aproximadamente 1 e indica que las soluciones se encuentran dentro del rango asintótico de convergencia.

\section{Estudio dependiente del tiempo}

A continuación, se presentan los resultados obtenidos durante la simulación realizada teniendo en cuenta el tiempo. Se presentan las ecuaciones que gobiernan el fenómeno y los rendimientos obtenidos

\section{Ecuaciones que gobiernan el fenómeno}

Debido a la naturaleza del fenómeno, es probable que las fuerzas viscosas infieran el comportamiento de los fluidos; por lo tanto, el sistema de ecuaciones RANS así como el modelo de turbulencia $k-\varepsilon$ (Chambergo, 2017) se usaron para calcular el momento y la transferencia de calor en los dominios Caughey (2014). Se usaron ecuaciones de continuidad y cinéticas para estimar las concentraciones de las especies.

$\frac{\partial \rho}{\partial t}+\nabla \cdot(\rho u)=0$

$\rho \frac{\partial u}{\partial t}+\rho(u \cdot \nabla) u=\nabla \cdot\left(-p 2 l+\left(\mu+\mu_{T}\right)\left(\nabla u+(\nabla u)^{T}\right)-\frac{2}{3}\left(\mu+\mu_{T}\right)(\nabla \cdot u) l-\frac{2}{3} \rho k l\right)+F$

$\rho \frac{\partial k}{\partial t}+\rho(u \cdot \nabla) k=\nabla \cdot\left(\left(\mu+\frac{\mu_{t}}{\sigma_{k}}\right) \nabla k\right)+P_{k}-\rho \epsilon$

$\rho \frac{\partial \epsilon}{\partial t}+\rho(u \cdot \nabla) \epsilon=\nabla \cdot\left[\left(\mu+\frac{\mu_{t}}{\sigma_{\epsilon}}\right) \nabla \epsilon\right]+C_{\epsilon(1)} \frac{\epsilon}{k} P k-C_{\epsilon(2)} \rho \frac{\epsilon^{2}}{k}, \epsilon=\mathrm{ep}$

$\mu_{T}=\rho C_{\mu} \frac{k^{2}}{\epsilon}$

$P_{k}=\mu_{T}\left[\nabla u:\left(\nabla u+(\nabla u)^{T}\right)-\frac{2}{3}(\nabla \cdot u)^{2}\right]-\frac{2}{3} \rho k \nabla \cdot u$

$\rho C_{P} \frac{\partial T^{2}}{\partial t}+\rho C_{P} u \cdot \nabla T 2=\nabla \cdot(k \nabla T 2)+Q$

$d X=d X\left(r_{b P}, \omega, t\right)$

$\frac{d \omega}{d t}=w$

$R_{i}=\nabla \cdot\left(-D_{i} \nabla C_{i}\right)+u \cdot \nabla C_{i}$

$N_{i}=-D_{i} \nabla C_{i}+u C_{i}$ 


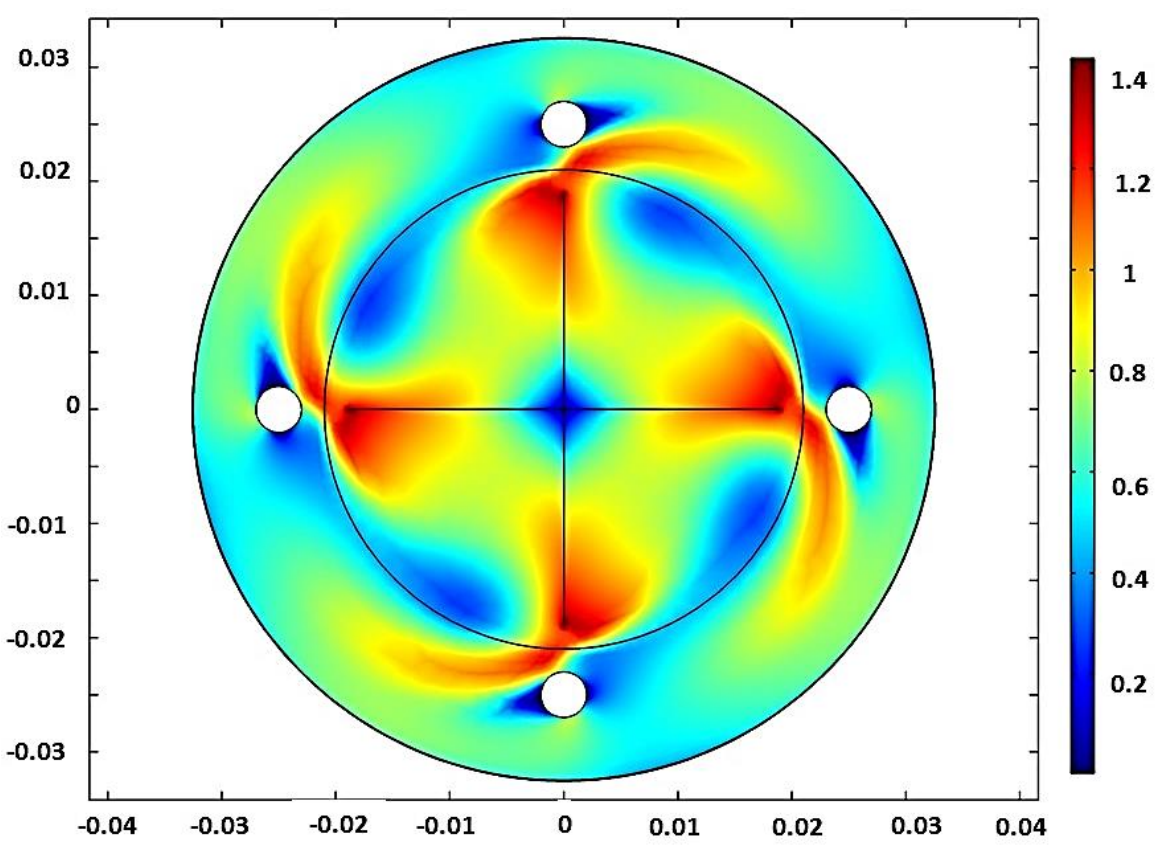

Fig.14: Perfil de velocidad (m/s) desarrollado para la malla \# 1

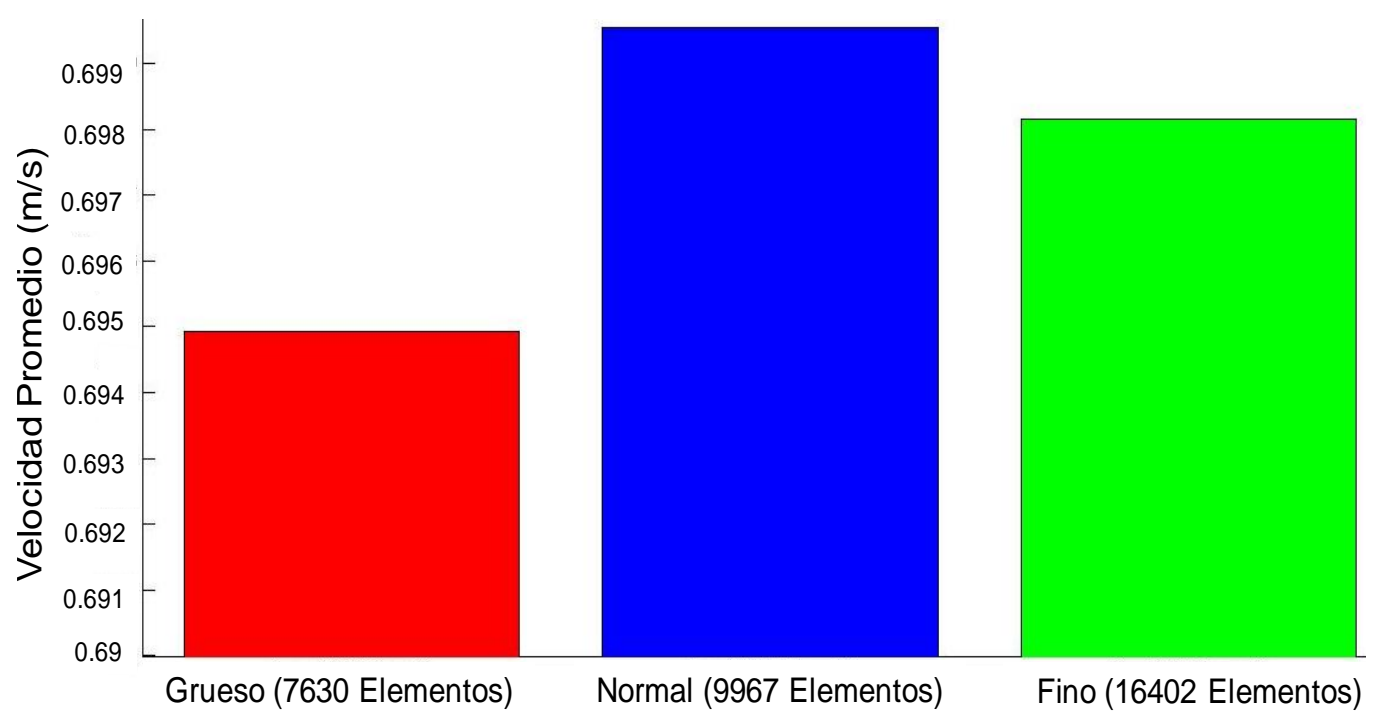

Fig.15: Comparación de la velocidad para cada malla

\section{Rendimientos obtenidos}

Como se mostró en la sección $\mathrm{OD}$, la elevación de temperatura tiene una influencia positiva en la producción de FA, en la simulación dinámica aparece un aumento de GAP entre los diferentes niveles de temperatura, por ejemplo, el rendimiento a $573 \mathrm{~K}$ y 2400 s $(40 \mathrm{~min}$ ) en este estudio es $20 \%$, mientras que anteriormente estaba cerca del $16 \%$, esto se puede explicar debido al aumento de contacto entre las moléculas causado por el movimiento de rotación inducido del impulsor.

\section{Concentración de ácidos grasos obtenidos}

El perfil de ácidos grasos en el área transversal se registró cada 10min (600s), la concentración inicial de FA fue de $346.3 \mathrm{~mol} \mathrm{~m}^{-3}$. Debido a que los perfiles presentan una forma muy similar entre las distintas temperaturas de trabajo, pero la magnitud es significativamente mayor para el perfil que corresponde a la temperatura de 573K. Los resultados registrados se pueden visualizar en la figura 16. 


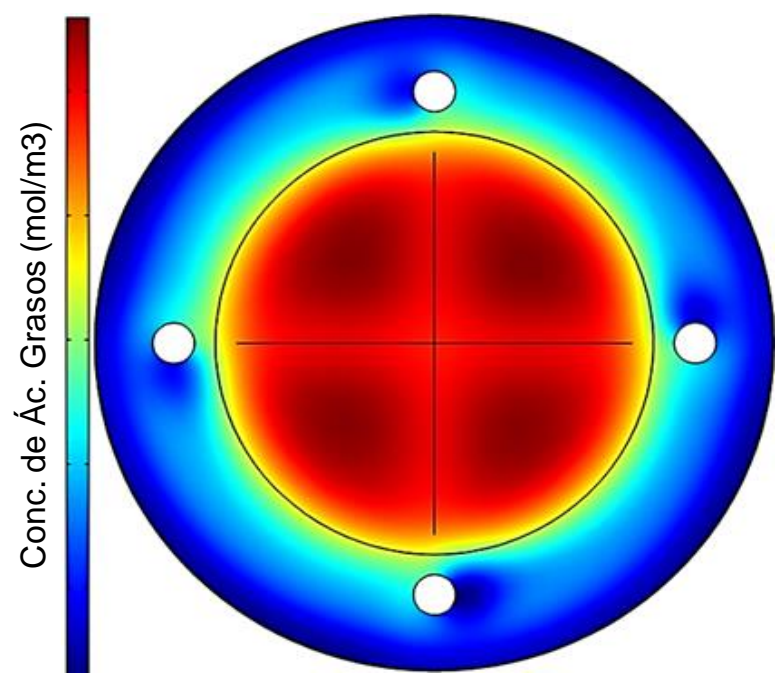

$\begin{array}{cc}\text { Temperatura }(\mathrm{K}) & \text { Escala }\left(\mathrm{mol} / \mathrm{m}^{3}\right) \\ 523 & {[570-590]} \\ 548 & {[525-545]} \\ 573 & {[595-615]}\end{array}$

Fig. 16: Perfil de concentración de FA a t=2400 s y $523 \mathrm{~K}, 548 \mathrm{~K}$ y $573 \mathrm{~K}$

\section{Comportamiento de la temperatura obtenida}

El perfil de distribución de temperatura es otro punto de comparación, que fue observado, entre los tratamientos de reacción, que ahora buscan uniformidad en lugar de magnitud. Las figuras 17, 18 y 19 confirman el impacto de tener el proceso a $573 \mathrm{~K}$, aumentar la temperatura de la fuente de calor ayuda a minimizar la propagación de la zona fría creada cerca del centro del impulsor.

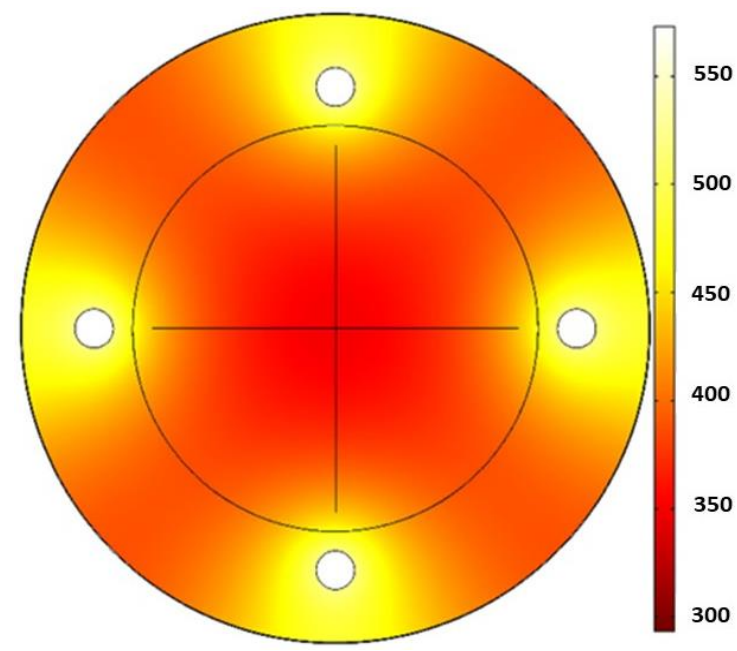

Fig 17: Perfil de Temperatura a $t=2400 \mathrm{~s}$

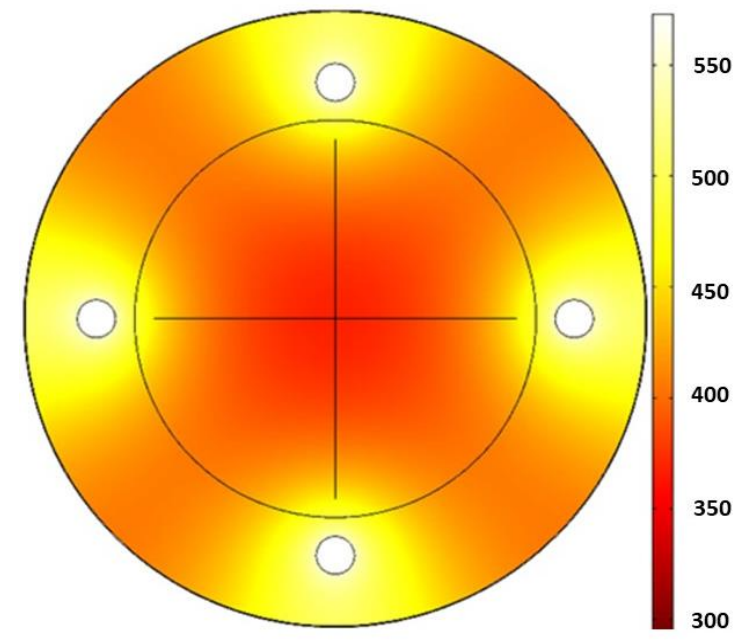

Fig. 18: Perfil de Temperatura FA a $\mathrm{t}=2400 \mathrm{~s}$

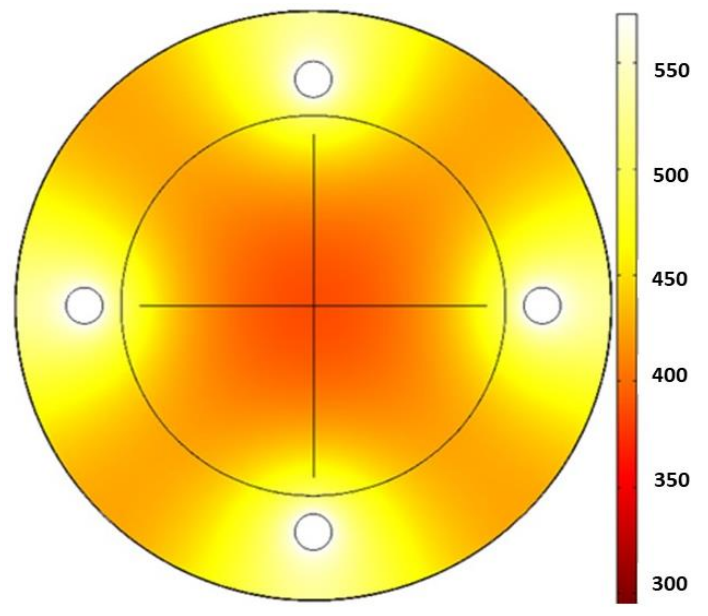

Fig. 19: Perfil de Temperatura a $\mathrm{t}=2400 \mathrm{~s}$ 


\section{CONCLUSIONES}

A partir de los resultados obtenidos, se plantean las siguientes conclusiones: 1) El estudio muestra que el programa COMSOL MULTIPHYSICS representa una buena herramienta para el modelamiento de este tipo de fenómenos; 2) mediante el uso de un modelado dividido en fases, que inicie con la generación del perfil de velocidades, se logra minimizar el costo computacional y no se tiene un efecto de distorsión en la simulación cinética en un dominio altamente turbulento; 3) si este tipo de modelado se complementa con una técnica de mallado MRF, se crea una reducción significativa en el uso del recurso computacional, lo que permite al usuario describir el fenómeno de manera más eficiente; 4) las diferencias entre los valores determinados en este trabajo y los reportados por Schall se pueden deber a un efecto de diferencias en la estimación de propiedades térmicas como la difusividad térmica y específica de calor de la mezcla de agua y aceite; 5) el resultado estimado a 548K fue muy similar al reportado por Schall, con un error aproximado del $4 \%$, lo que lleva a concluir que este modelo representa de buena manera ese comportamiento.

\section{RECOMENDACIONES}

Se sugiere realizar simulaciones con geometrías asimétricas, por ejemplo, desalinear el impeller con respecto a los calentadores con el fin de evaluar los gradientes de velocidad.

\section{AGRADECIMIENTOS}

Los autores agradecen a la Universidad del Norte y a las empresas Palmaceite S.A y Aceites S.A, el apoyo y las facilidades otorgadas para la realización de este artículo.

\section{NOTACIÓN}

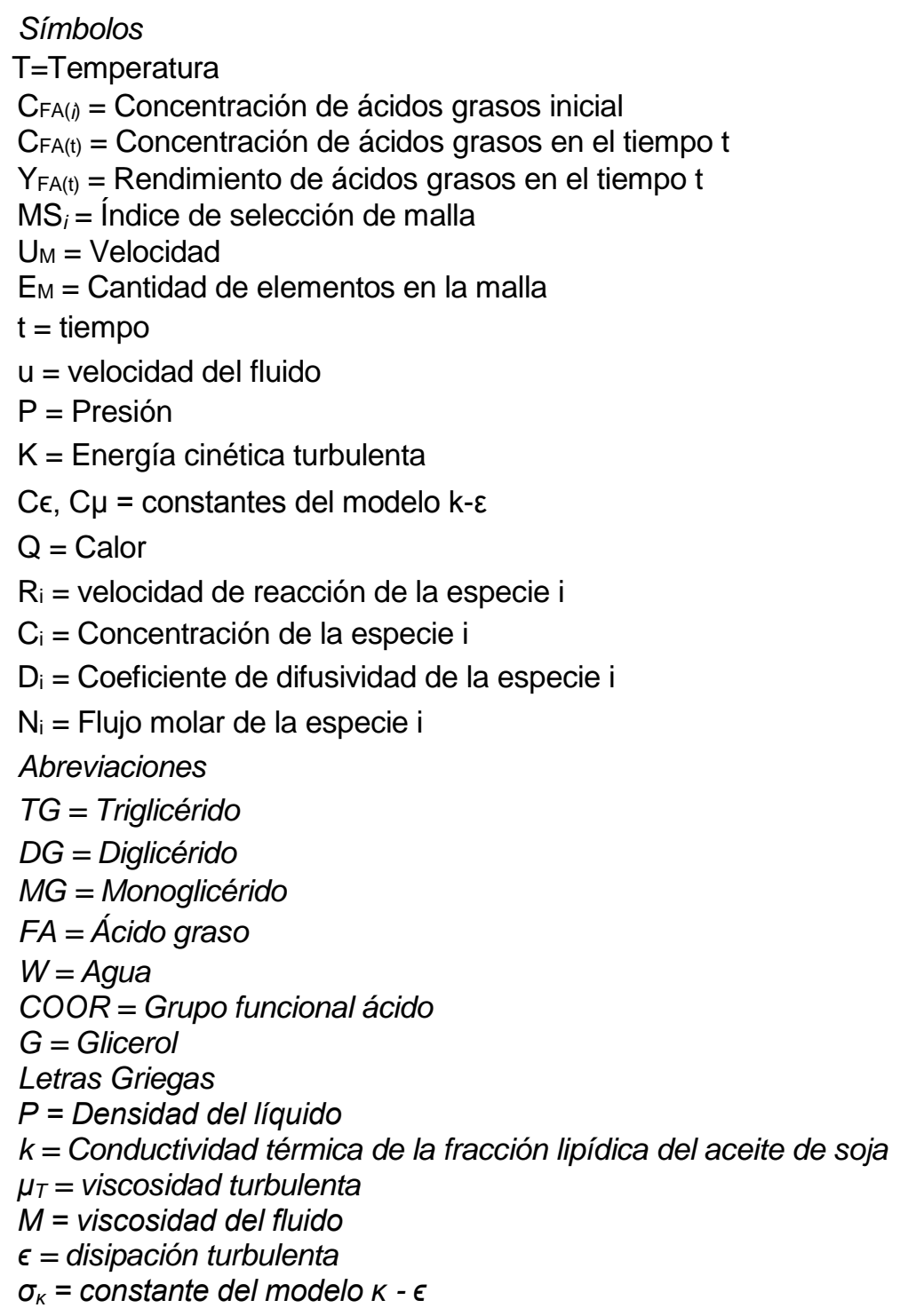




\section{REFERENCIAS}

Abdelmoez, W., S. Nage, A. Bastawess, A. Ihab y H. Yoshida, Subcritical Water Technology for Wheat Straw Hydrolysis to Produce Value Added Products, doi:10.1016/j.jclepro.2014.02.011, Journal of Cleaner Production, 70, 68-77 (2014)

Alenezi, R., G. Leeke, R. Santos y A. Khan, Hydrolysis Kinetics of Sunflower Oil under Subcritical Water Conditions, doi:10.1016/j.cherd.2008.12.009, Chemical Engineering Research and Design, 87(6), 867-873 (2009)

Baldyga, J., M. Henczka y L. Makowski, $4^{\text {th }}$ International Symposium on Mixing in Industrial Processes: Effects of Mixing on Parallel Chemical Reactions in a Continuous-Flow Stirred-Tank Reactor, doi:10.1205/02638760152721109, Chemical Engineering Research and Design, 79(8), 895-900 (2001)

Bandyopadhyay, S. y R. Bhattacharya, Discrete and Continuous Simulation: Theory and Practice, $1^{\text {a }}$ Ed., CRC Press, Florida, EE.UU (2014)

Barbosa-Canovas, G. y A. Ibarz, Unit Operations in Food Engineering, CRC Press, Florida, EE.UU, p. 312 (2002)

Carr, A., R. Mammucari y N. Foster, A Review of Subcritical Water as a Solvent and its Utilisation for the Processing of Hydrophobic Organic Compounds, doi:10.1016/j.cej.2011.06.007, Chemical Engineering Journal, 172(1), 1-17 (2011)

Caughey, D., Computational Fluid Dynamics, Computer Science and Software Engineering (2014)

Cambergo, J., Q. Valverde, A. Pachas y H. Yépez, Estudio del Comportamiento Fluido-dinámico de un Agitador a Escala Reducida mediante Simulación Numérica, doi: 10.4067/S0718-07642017000300005, Información Tecnológica, 28(03), 37-46 (2017)

Conelly, R. y J. Kokini, Analysis Mixing Processes Using CFD. In D.-W. Sun (Ed.), Computational Fluid Dynamics in Food Processing, 555-587 (2007)

Diasakou, M., A. Louloudi y N. Papayannakos, Kinetics of the Non-Catalytic Transesterification of Soybean Oil, doi:10.1016/S0016-2316(98)00025-8, Fuel, 77(12), 1297-1302 (1998)

Dijkstra, A., Soybean Oil, In Encyclopedia of Food and Health, 58-63, Oxford, Academic Press (2016)

Erhan, S. y J. Perez, Biobased Industrial Fluids and Lubricants, AOCS Publishing (2002)

Hongna, W., J. Xiaogiang, W. Xue, Z. Zhengxi, W. Jianping y Z. Jinli, CFD Modeling of Hydrodynamic Characteristics of a Gas Liquid Two Phase Stirred Tank, doi:10.1016/j.apm.2013.05.032, Applied Mathematical Modelling, 38(1), 63-92 (2014)

Milliren, A., J. Wissinger, V. Gottumukala y C. Schall, Kinetics of Soybean Oil Hydrolysis in Subcritical Water, doi:10.1016/j.fuel.2012.12.068, Fuel, 108, 277-281 (2013)

Pinto, J. y F. Lancas, Hydrolysis of Corn Oil Using Subcritical Water, doi:10.1590/S0103-50532006000100013, Journal of the Brazilian Chemical Society, 17(1), 87-89 (2006)

Plaza, M. y C. Turner, Pressurized Hot Water Extraction of Bioactives, doi:10.1016/j.trac.2015.02.022, TrAC Trends in Analytical Chemistry, 71, 39-54 (2015)

Pramote, K., F. Tomoyuki, A. Shuji, K. Yukitaka y M. Ryuichi, Kinetics on the Hydrolysis of Fatty Acid Esters in Subcritical Water, doi:10.1016/j.cej.2003.08.002, Chemical Engineering Journal, 99(1), 1-4 (2004)

Pramote, K., F. Tomoyuki, A. Shuji, K. Yukitaka y M. Ryuichi, Kinetics on the Hydrolysis of Fatty Acid Esters in Subcritical Water, doi:10.1016/j.cej.2003.08.002, Chemical Engineering Journal, 99(1), 1-4 (2004)

Qin, M., F. Xiao-Dan, L. Xiao-Cao, Q. Tai-Qiu y J. Jian-Guo, Ultrasound-Enhanced Subcritical Water Extraction of Essential Oils from Kaempferia Galangal L. and their Comparative Antioxidant Activities, doi:10.1016/j.seppur.2015.06.013, Separation and Purification Technology, 150, 73-79 (2015)

Reddy, H., T. Muppaneni, Y. Sun, Y. Li, S. Ponnusamy, P. Patil y S. Deng, Subcritical Water Extraction of Lipids from Wet Algae for Biodiesel Production, doi: 10.1016/j.fuel.2014.04.081, Fuel, 133, 73-81 (2014)

Roache,-Patrick J., Quantification of Uncertainty in Computational Fluid Dynamics, Annual review of fluid Mechanics, 29, 123-160 (1997)

Rudniak, L., P. Machniewski y A. Molga, CFD Modelling of Stirred Tank Chemical Reactors: Homogeneous and Heterogeneous Reaction Systems, doi:10.1016/j.ces.2004.09.014, Chemical Engineering Science, 59(2223), 52335239 (2004)

Ting, C. y C. Chen, Viscosity and Working Efficiency Analysis of Soybean Oil Based Bio-Lubricants, doi:10.1016/j.measurement.2011.04.005, Measurement, 44(8), 1337-1341 (2011)

Wang, W., R. Natelson, L. Stikeleather y W. Roberts, CFD Simulation of Transient Stage of Continuous Countercurrent Hydrolysis of Canola Oil, doi:10.1016/j.compchemeng.2012.04.008, Computers and Chemical Engineering, 43(10), 108119 (2012)

Wolfe, N. y P. Jerrers, Handbook of Property Estimation Methods for Chemicals Environmental Health Sciences, (Robert S. Ed.), Boethling CRC Press (2000) 
